ARTICLE

https://doi.org/10.1038/s41467-019-10104-4

\title{
Strategic-tuning of radiative excitons for efficient and stable fluorescent white organic light-emitting diodes
}

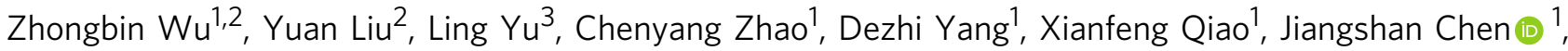 \\ Chuluo Yang ${ }^{3}{ }^{3}$, Hans Kleemann ${ }^{2}$, Karl Leo ${ }^{2} \&$ Dongge $\mathrm{Ma}^{1}$
}

The emerging thermally activated delayed fluorescence materials have great potential for efficiencies in organic light-emitting diodes by optimizing molecular structures of the emitter system. However, it is still challenging in the device structural design to achieve high efficiency and stable device operation in white organic light-emitting diodes. Here we propose a universal design strategy for thermally activated delayed fluorescence emitter-based fluorescent white organic light-emitting diodes, establishing an advanced system of "orange thermally activated delayed fluorescence emitter sensitized by blue thermally activated delayed fluorescence host" combined with an effective exciton-confined emissive layer. Compared to reference single-layer and double-layer emissive devices, the external quantum efficiency improves by 31 and 45\%, respectively, and device operational stability also shows nearly fivefold increase. Additionally, a detailed optical simulation for the present structure is made, indicating the validity of the design strategy in the fluorescent white organic lightemitting diodes.

\footnotetext{
${ }^{1}$ Institute of Polymer Optoelectronic Materials and Devices, State Key Laboratory of Luminescent Materials and Devices, South China University of Technology, Guangzhou 510640, People's Republic of China. ${ }^{2}$ Dresden Integrated Center for Applied Physics and Photonic Materials (IAPP), Technische Universität Dresden, Nöthnitzer Str. 61, Dresden 01187, Germany. ${ }^{3}$ Hubei Collaborative Innovation Center for Advanced Organic Chemical Materials, Hubei Key Lab on Organic and Polymeric Optoelectronic Materials, Department of Chemistry, Wuhan University, Wuhan 430072, People's Republic of China. Correspondence and requests for materials should be addressed to K.L. (email: karl.leo@iapp.de) or to D.M. (email: msdgma@scut.edu.cn)
} 
W hite organic light-emitting diodes (WOLEDs) have attracted substantial attention nowadays due to their promising merits in solid-state lighting sources and full-color displays ${ }^{1-3}$. Enormous efforts have been devoted to efficient WOLEDs, such as employing the precise doping concentration regulation strategy ${ }^{4-8}$, eliminating the inserting layer between fluorescent and phosphorescent emissive regions ${ }^{9}$, utilizing exciplex systems ${ }^{10,11} ; \quad \mathrm{p}-\mathrm{i}-\mathrm{n}$ junctions ${ }^{12} ;$ tandem structure $^{13,14}$; down-conversion ${ }^{15}$; exciton-confining structures ${ }^{16}$, and introducing the external heavy-atom effect ${ }^{17}$. According to the spin statistics, the singlet and triplet excitons can be generated by a 1:3 ratio in organic materials when the holes and electrons recombine $^{18,19}$. As seen, the key to realize highly efficient WOLEDs is that all electrically generated excitons need to be effectively used for emission. For all phosphorescent WOLEDs, owing to the strong spin-orbit coupling, all singlet and triplet excitons can be harvested, achieving unity exciton utilization efficiency, thus the phosphorescent WOLEDs have been considered as the most promising device architecture during the past years ${ }^{20}$. But there is still a bottleneck to simultaneously realize high efficiency and long device lifetime in phosphorescent WOLEDs, which is restricted by the absence of the efficient and stable blue phosphorescent emitters. Then, hybrid WOLEDs combining the stable blue fluorophores and efficient longwavelength phosphores can likewise harvest all generated excitons by the "triplet harvesting" strategy, yielding the compatibility of good stability and high efficiency ${ }^{21-24}$.

Recently, $E$-type delayed fluorescence ${ }^{25}$, i.e., thermally activated delayed fluorescence (TADF) without the metal-organic complexes has aroused researchers' interest, which has been widely considered as a promising route to harvest nonradiative triplet excitons in OLEDs ${ }^{26}$. Because the energy gap between the singlet excited state and triplet excited state is sufficiently small in TADF processes, the generated nonradiative triplet excitons can be efficiently upconverted into the radiative singlet excitons by the reverse intersystem crossing (RISC) process ${ }^{27-31}$. TADFbased monochrome OLEDs employing pure organic aromatic compounds as emitters have realized nearly $100 \%$ exciton production efficiency and the maximum external quantum efficiency (EQE) has reached beyond 30\%, and meanwhile the operational stability has improved so much ${ }^{32,33}$, but it is still unsatisfactory in the development of TADF-based WOLEDs. Several preliminary studies have been reported on the all-fluorescent WOLEDs. Adachi's group ${ }^{34}$ pioneered TADF emitter-based fluorescent WOLEDs with a maximum EQE of $6.7 \%$ and current efficiency (CE) of $16.7 \mathrm{~cd} \mathrm{~A}^{-1}$. Then, they introduced a high-energy-level blue TADF molecule as the common host and conventional red/ green fluorescent molecules as dopants, a maximum EQE of $12.1 \%$ was realized ${ }^{35}$. Zhao et al. ${ }^{36}$ demonstrated fluorescent WOLEDs composed of a blue TADF host and an orange fluorescent dopant with the maximum EQE of 7.48\%, CE of 20.2 $\mathrm{cd} \mathrm{A}^{-1}$, power efficiency (PE) of $15.9 \mathrm{~lm} \mathrm{~W}^{-1}$. Lee et al. ${ }^{37}$ doped two conventional fluorescent emitters into a TADF host to form a single-emitting layer (EML) fluorescent white device with a maximum EQE of $14.0 \%$ and a maximum $\mathrm{PE}$ of $36.2 \mathrm{~lm} \mathrm{~W}^{-1}$. Recently, Su et al. designed an efficient yellow TADF emitter combined with a blue TADF emitter (3,6-2TPA-TX). They achieved a maximum EQE of $20.4 \%$ in their fluorescent WOLED using common multi-EML structures ${ }^{38}$. Despite of these progress, efficient and stable TADF-based WOLEDs have not ever been achieved by adequate utilization of triplet excitons and tailored device design. Actually, it is really challenging to develop efficient and stable device design strategies for all-fluorescent WOLEDs that can render various channels to harvest all singlet and triplet excitons and achieve balanced charge injection as well as effective exciton and charge carrier confinement.
Here, we show an efficient and stable strategy using an orange TADF emitter sensitized by a high-energy-level blue TADF host. The key point is doping an orange TADF emitter with an ultralow concentration into a blue TADF emissive host, forming a doubledopant system combined with an effective exciton-confined emissive layer architecture to fully utilize all electrically generated excitons. Using this approach, extremely high-efficiency fluorescent WOLEDs with nearly $100 \%$ exciton utilization efficiency can be realized. The resulting WOLED exhibits excellent electroluminescence (EL) performance with the maximum EQE, CE, and $\mathrm{PE}$ of $20.5 \%, 51.3 \mathrm{~cd} \mathrm{~A}^{-1}$, and $59.6 \mathrm{~lm} \mathrm{~W}^{-1}$, respectively, which should be among the highest values for all-fluorescent WOLEDs. The device also shows very impressive operational stability by the proposed strategy. We also present a comprehensive optical simulation for our WOLEDs. These results indicate that proper device architecture facilitates highly effective harvesting of all generated singlet and triplet excitons in TADFbased fluorescent WOLEDs.

\section{Results}

Construction of the device structure. To illustrate our device design strategy, the selection of emitting dopants is a primary consideration. When the TADF-emitting dopants with a very small energy gap between $S_{1}$ and $T_{1}$ are used to fabricate OLEDs, the internal quantum efficiency (IQE) can be expressed as,

$$
\eta_{\mathrm{IQE}}=\left[0.25+\frac{\left(0.25 \phi_{\mathrm{ISC}, \mathrm{D}}+0.75\right) \phi_{\mathrm{RISC}}}{1-\phi_{\mathrm{ISC}, \mathrm{D}} \phi_{\mathrm{RISC}}}\right] \phi_{\mathrm{PL}, \mathrm{D}}
$$

where $\phi_{\mathrm{ISC}, \mathrm{D}}$ is the ISC efficiency, $\phi_{\mathrm{RISC}}$ is the RISC efficiency, $\phi_{\mathrm{PL}}$ ${ }_{D}$ is the PL quantum yield of the dopants. Therein, $\phi_{I S C, D}$ and $\phi_{\text {RISC }}$ can be calculated as follows,

$$
\begin{gathered}
\phi_{\mathrm{ISC}, \mathrm{D}}=\frac{\kappa_{\mathrm{ISC}}}{\kappa_{\mathrm{ISC}}+\kappa_{\mathrm{r}}+\kappa_{\mathrm{nr}}} \\
\phi_{\mathrm{RISC}}=\frac{\kappa_{\mathrm{RISC}}}{\kappa_{\mathrm{RISC}}+\kappa_{\mathrm{nr}}^{\mathrm{T}}}
\end{gathered}
$$

where $\kappa_{\mathrm{nr}}$ is the nonradiative decay rate of the singlets and $\kappa_{\mathrm{nr}}^{\mathrm{T}}$ is the rate for the triplet decay processes, except for RISC. As can be seen, the $\phi_{\mathrm{RISC}} / \phi_{\mathrm{ISC}, \mathrm{D}}$ and $\phi_{\mathrm{PL}}$ of the dopants determine IQE. Thus, TADF materials with high $\phi_{\mathrm{RISC}} / \phi_{\mathrm{ISC}, \mathrm{D}}$ and $\phi_{\mathrm{PL}}$ values as the emitters are necessary to realize efficient fluorescent WOLEDs. In this work, bis[4-(9,9-dimethyl-9,10-dihydroacridine)phenyl]sulfone (DMAC-DPS) for blue emission ${ }^{39}$ and 10-(7-fluoro-2,3-diphenylquinoxalin-6-yl)-10H-phenoxazine (FDQPXZ) for orange emission with high $\phi_{\mathrm{RISC}} / \phi_{\mathrm{ISC}, \mathrm{D}}$ and $\phi_{\mathrm{PL}}$ values $^{40}$ are selected as the emitting dopants. Bis(2-(diphenylphosphino)phenyl)ether oxide (DPEPO) with a high $\mathrm{T}_{1}$ level of $3.30 \mathrm{eV}$ acts as the host of DMAC-DPS. The well-known ambipolar material 4,4'-bis(N-carbazolyl)biphenyl (CBP) is utilized as the host for the orange emitter. To minimize the working voltage and improve the charge balance, $20 \mathrm{wt} \%$ hexaazatriphenylene hexacarbonitrile (HATCN) doped into 1,1'-bis[4-(di-p-tolylamino)phenyl]cyclohexane (TAPC) and $3 \mathrm{wt} \%$ lithium carbonate $\left(\mathrm{Li}_{2} \mathrm{CO}_{3}\right)$ doped into 1,3-bis(3,5-dipyrid-3-yl-phenyl)benzene $(\mathrm{BmPyPB})$ are used as the hole-transporting and electrontransporting layers, respectively.

Device structure and performance. Figure 1 shows illustrated energy-level diagram and chemical structures of the used materials. It is noteworthy that TAPC and BmPyPB can effectively confine holes and electrons within the exciton recombination zone. On the one hand, the utilization of TAPC could prevent electrons transporting across the emissive layer, thus, substantially minimizing the electron leakage from the emissive layer 
a
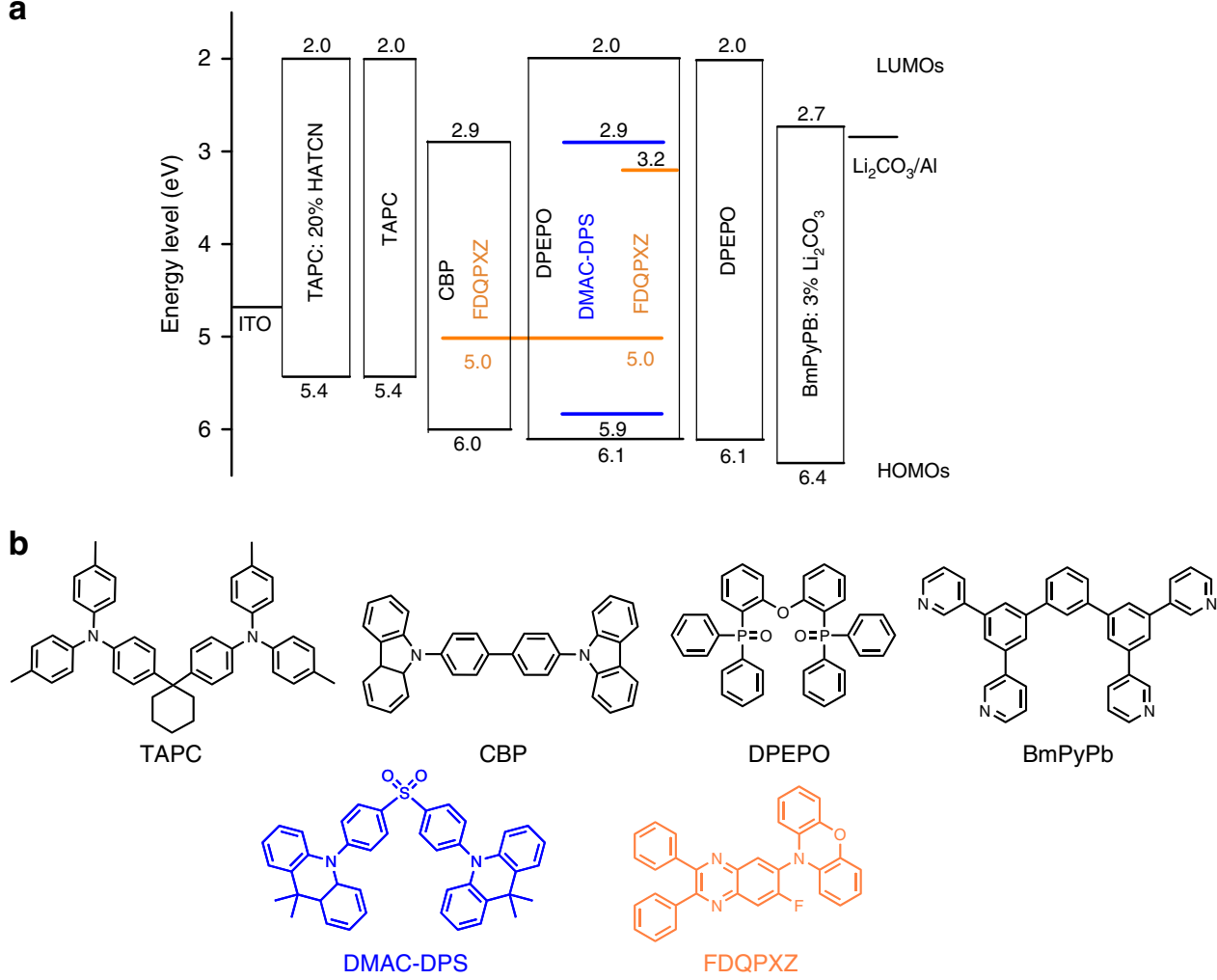

Fig. 1 Energy-level diagram and chemical molecular structures. a Proposed energy-level diagram of the fluorescent white organic light-emitting diodes (WOLEDs). Note that these numbers indicate the respective highest occupied and lowest unoccupied molecular orbital (HOMO and LUMO, respectively) energies relative to the vacuum level. $\mathbf{b}$ Chemical molecular structures of the functional materials used

because of the smaller lowest unoccupied molecular orbital (LUMO) of TAPC than that of CBP and FDQPXZ. On the other hand, we also notice that there is the energy offset $(0.3 \mathrm{eV})$ of the highest unoccupied molecular orbital (HOMO) between BmPyPB and DPEPO, which will prevent the injected hole transport into the nonradiative layer, thus, effectively confines well the holes in the emissive layer. The detailed design produces an energetic well-like emissive zone. When the injected charges or generated excitons enter into the emissive region, they would not leak out or get back, increasing the likelihood that singlet and triplet excitons will radiatively decay within the emissive zone.

After optimizing the doping concentration and emissive layer thickness, our final structure is ITO/HATCN (10 nm)/TAPC: 20 wt $\%$ HATCN $(50 \mathrm{~nm}) /$ TAPC $(20 \mathrm{~nm}) / \mathrm{CBP}: 3 \mathrm{wt} \%$ FDQPXZ (10 nm)/DPEPO: $30 \mathrm{wt} \%$ DMAC-DPS: $0.5 \mathrm{wt} \%$ FDQPXZ $(6 \mathrm{~nm}) /$ DPEPO $(10 \mathrm{~nm}) / \mathrm{BmPyPB}: 3 \mathrm{wt} \% \mathrm{Li}_{2} \mathrm{CO}_{3}(50 \mathrm{~nm}) / \mathrm{Li}_{2} \mathrm{CO}_{3} \quad(1$ $\mathrm{nm}) / \mathrm{Al}(100 \mathrm{~nm})$. Figure $2 \mathrm{a}$ illustrates the proposed exciton energy-transfer processes within the emissive zone (also shown in Supplementary Fig. 1), which will be proved below. Due to the electron-transporting property of DPEPO and the superior ambipolar-transporting characteristics of $\mathrm{CBP}^{41}$, it can be expected that the main recombination zone should be across the interface of CBP:3\% FDQPXZ and DPEPO:30\% DMACDPS:0.5\% FDQPXZ, and the broadening exciton recombination zone also benefits the improvement of efficiency roll-off. On the basis of this structural engineering via an orange TADF emitter sensitized by a high-energy-level blue TADF host, the resulting WOLED exhibits very impressive EL performances, as shown in Fig. 2b and Supplementary Fig. 2. The maximum forwardviewing EQE, CE, and PE reach 20.5\%, $51.3 \mathrm{~cd} \mathrm{~A}^{-1}$, and $59.6 \mathrm{~lm}$ $\mathrm{W}^{-1}$, respectively. At a typical display luminance of $100 \mathrm{~cd} \mathrm{~m}^{-2}$, they remain as high as $18.8 \%, 46.5 \mathrm{~cd} \mathrm{~A}^{-1}$, and $52.4 \mathrm{~lm} \mathrm{~W}^{-1}$. All the results are obtained in the forward direction without any outcoupling techniques. Table 1 summarizes key performance parameters of the resulting WOLEDs. Figure $2 \mathrm{c}$ reveals a low turn-on voltage of $2.6 \mathrm{~V}$ and a rather low-driving voltage of $3.2 \mathrm{~V}$ at the brightness of $1000 \mathrm{~cd} \mathrm{~m}^{-2}$. In addition, the EL spectra cover all wavelengths from 400 to $780 \mathrm{~nm}$ (see the inset of Fig. 2b), and the CRI is calculated to reach 72. Notably, this WOLED exhibits high stability of the EL spectra. Varying the brightness from 100 to $5000 \mathrm{~cd} \mathrm{~m}^{-2}$ (corresponding to the driving voltage of 3-4.2 V), the WOLED shows negligible alteration of the 1931 Commission Internationale de L'Eclairage (CIE) coordinates, CIE $x=0.323-0.330$ and CIE $y=0.411-0.416$, thus revealing superior chromatic stability.

To compare with WOLEDs with conventional emissive structures, we also optimized fluorescent WOLEDs using the conventional single-emissive layer and double-emissive layer structure as the reference devices. The single-emissive layer fluorescent WOLED is ITO/HATCN (10 nm)/TAPC:20 wt $\%$ HATCN $(50 \mathrm{~nm}) / \mathrm{TAPC}(20 \mathrm{~nm}) / \mathrm{DPEPO}: 25 \mathrm{wt} \%$ DMAC-DPS: 1 wt $\%$ FDQPXZ $(20 \mathrm{~nm}) / \mathrm{DPEPO}(10 \mathrm{~nm}) / \mathrm{BmPyPB}: 3 \mathrm{wt} \% \mathrm{Li}_{2} \mathrm{CO}_{3}$ $(50 \mathrm{~nm}) / \mathrm{Li}_{2} \mathrm{CO}_{3}(1 \mathrm{~nm}) / \mathrm{Al}(100 \mathrm{~nm})$, and the conventional double-emissive layer with separated blue and orange emissive layers has the structure of ITO/HATCN (10 nm)/TAPC:20 wt\% HATCN $(50 \mathrm{~nm}) / \mathrm{TAPC}(20 \mathrm{~nm}) / \mathrm{CBP}: 3 \mathrm{wt} \%$ FDQPXZ $(10 \mathrm{~nm}) /$ DPEPO: $25 \mathrm{wt} \%$ DMAC-DPS (10 nm)/DPEPO $(10 \mathrm{~nm}) /$ BmPyPB:3 wt $\% \mathrm{Li}_{2} \mathrm{CO}_{3}(50 \mathrm{~nm}) / \mathrm{Li}_{2} \mathrm{CO}_{3}(1 \mathrm{~nm}) / \mathrm{Al} \quad(100 \mathrm{~nm})$. Their EL performance was shown in Fig. 3 and Supplementary Fig. 2. The single-emissive layer and double-emissive layer fluorescent WOLEDs realized the maximum EQEs of $14.1 \%$ and $15.7 \%$, CEs of $39.4 \mathrm{~cd} \mathrm{~A}^{-1}$ and $39.1 \mathrm{~cd} \mathrm{~A}^{-1}$, PEs of $45.8 \mathrm{~lm}$ $\mathrm{W}^{-1}$ and $41.0 \mathrm{~lm} \mathrm{~W}^{-1}$, respectively. Obviously, we can see that our proposed emissive layer with the orange TADF emitter 

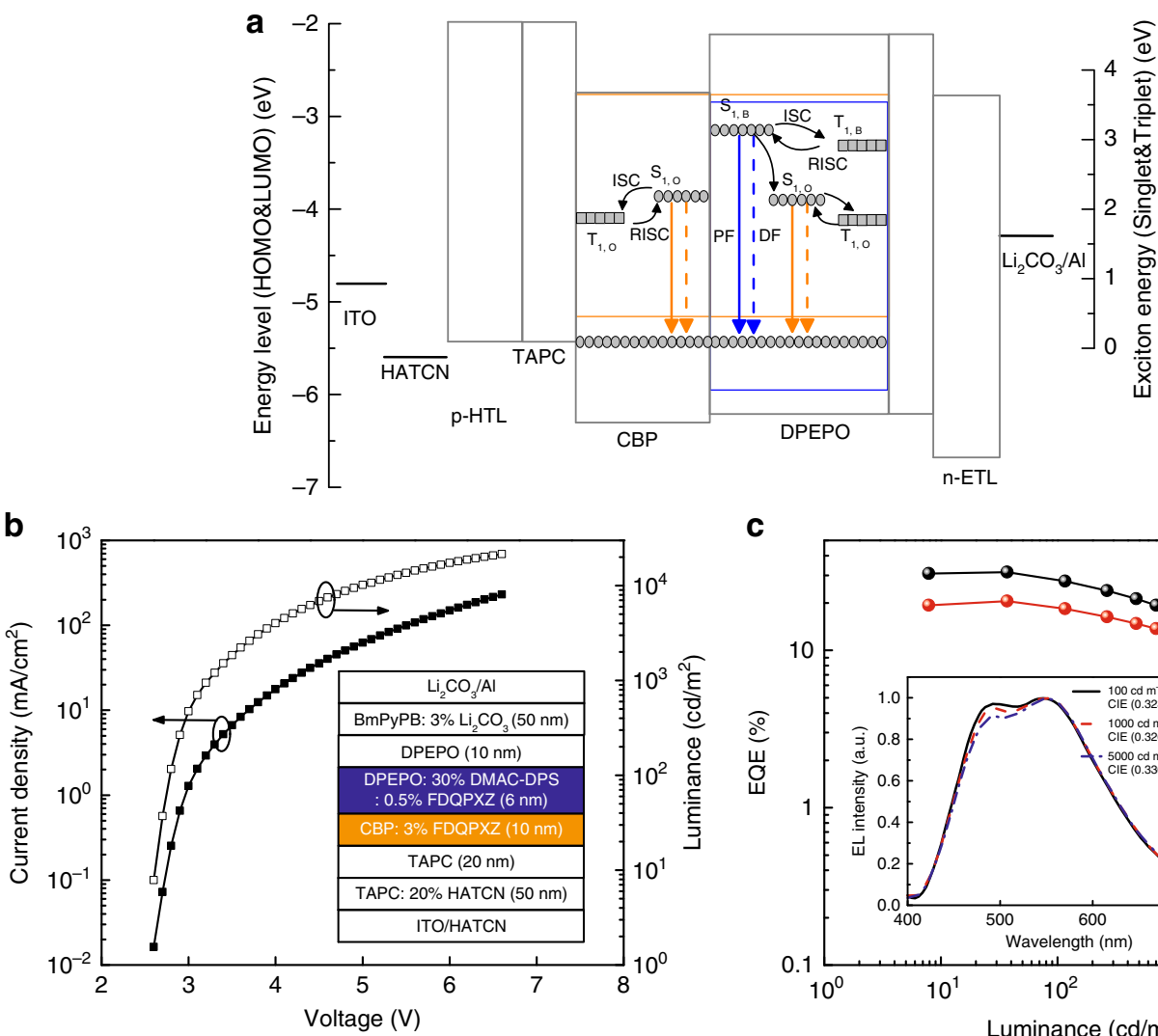

C
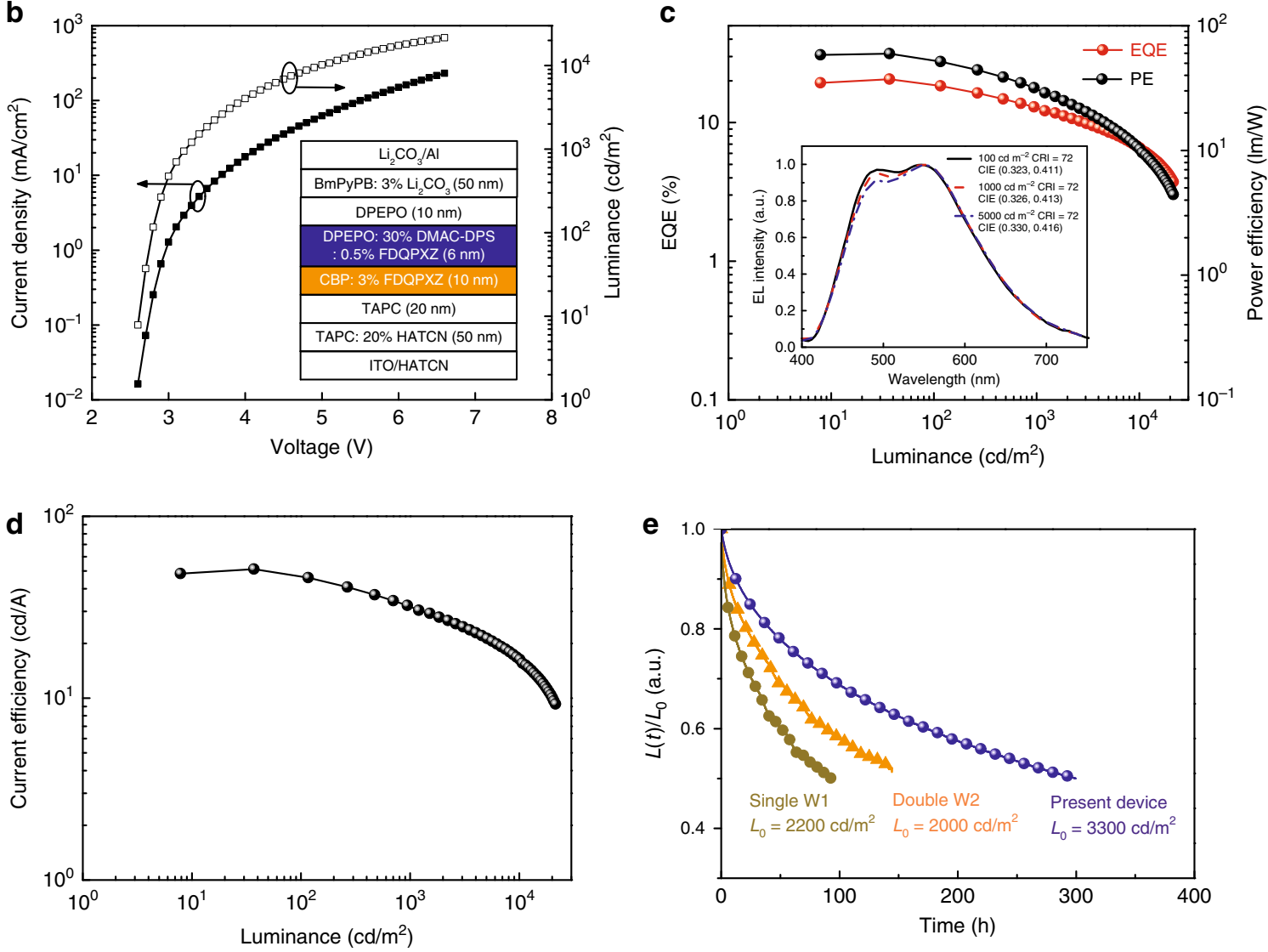

Fig. 2 Energy transfer processes and device performance. a Energy transfer processes in the emissive zones of the optimized fluorescent white organic light-emitting diodes (WOLEDs). b Current density-voltage-luminance $(J-V-L)$ characteristics of the fluorescent WOLEDs. The inset illustrates the detailed device structure of the optimized WOLEDs. c External quantum efficiency (EQE) and power efficiency (PE)-luminance characteristics of the fluorescent WOLED. Inset exhibits the normalized electroluminescence spectra at the different luminance of 100,1000 . and $5000 \mathrm{~cd} / \mathrm{m}^{2}$, and the respective Commission Internationale de L'Eclairage (CIE) and color rendering index (CRI). d Current efficiency-luminance characteristics of present WOLED. e Device lifetime measurement of conventional single- and double-emissive layer WOLEDs, and the present WOLED with proposed emissive architecture

sensitized by a blue TADF host shows much higher device performance, the maximum EQEs improve by 45 and $31 \%$, respectively. Although the reference device with the singleemissive layer also exhibits this sensitized effect, the generated excitons in the EML can leak into the adjacent TAPC layer without the effective exciton limitation owing to the high-energy level of DPEPO, resulting in the low exciton utilization efficiency. Besides, the main exciton recombination zone in the reference device locates in a very narrow region near the interface of TAPC/ EML because of the strong unipolar electron-transporting property of DPEPO, which would lead to the severe exciton quenching at high current density.
Our design strategy provides not only obvious increase of device efficiency but also a significant enhancement of operational stability in the present WOLEDs. For example, the normalized luminance of the fluorescent WOLEDs as a function of operation time at initial luminance are presented in Fig. 2e, the lifetime $\left(\mathrm{T}_{50}\right)$, defined as the elapsed operation time at which the luminance drops to $50 \%$ of the initial value, is about $300 \mathrm{~h}$ for our present device at initial luminance of $3300 \mathrm{~cd} \mathrm{~m}^{-2}$. To predict $\mathrm{T}_{50}$ at $L_{0}=1000 \mathrm{~cd} \mathrm{~m}^{-2}$, an acceleration factor of 1.7 for each device from the lifetime measurements can be obtained. According to $\left(L_{0}\right)^{\mathrm{n}} \times \mathrm{T}_{50}=$ constant $^{42,43}, \mathrm{~T}_{50}=2283 \mathrm{~h}$ for our present device, $382 \mathrm{~h}$ for single $\mathrm{W} 1$ and $487 \mathrm{~h}$ for double W2 at the initial luminance of $1000 \mathrm{~cd} \mathrm{~m}^{-2}$. As seen, the lifetime of our 
Table 1 Summary of device performances

\begin{tabular}{|c|c|c|c|c|c|c|c|c|c|}
\hline \multirow[t]{2}{*}{ Device } & \multirow[t]{2}{*}{$V_{\text {on }}{ }^{a}(V)$} & \multirow[t]{2}{*}{$\begin{array}{l}\text { Max } \\
\text { EQEb [\%] }\end{array}$} & \multirow[t]{2}{*}{$\begin{array}{l}\text { Max CEb } \\
{\left[c d A^{-1}\right]}\end{array}$} & \multirow[t]{2}{*}{$\begin{array}{l}\text { Max PEb } \\
{\left[\operatorname{lm} W^{-1}\right]}\end{array}$} & \multirow[t]{2}{*}{ CRIC } & \multirow[t]{2}{*}{$\operatorname{CIE}^{c}(x, y)$} & \multicolumn{3}{|c|}{$\begin{array}{l}\text { Performance at the brightness of } \\
100 / 1000 \mathrm{~cd} \mathrm{~m}^{-2}\end{array}$} \\
\hline & & & & & & & EQE [\%] & $C E\left[c d A^{-1}\right]$ & PE $\left[\operatorname{Im~} \mathbf{W}^{-1}\right]$ \\
\hline Double-EML & 2.8 & 15.7 & 39.1 & 41.0 & 70 & $(0.322,0.414)$ & $12.3 / 9.3$ & $30.4 / 23.0$ & $28.4 / 17.3$ \\
\hline Proposed EML & 2.6 & 20.5 & 51.3 & 59.6 & 72 & $(0.326,0.413)$ & $18.8 / 13.0$ & $46.5 / 31.9$ & $52.4 / 31.7$ \\
\hline
\end{tabular}
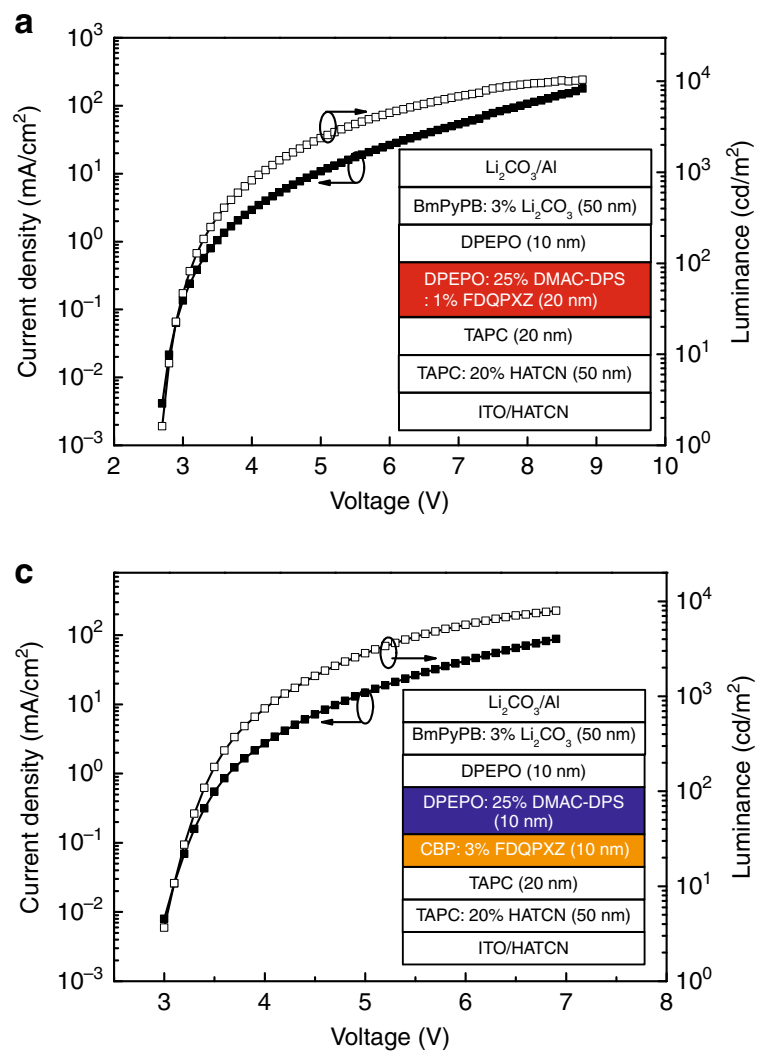
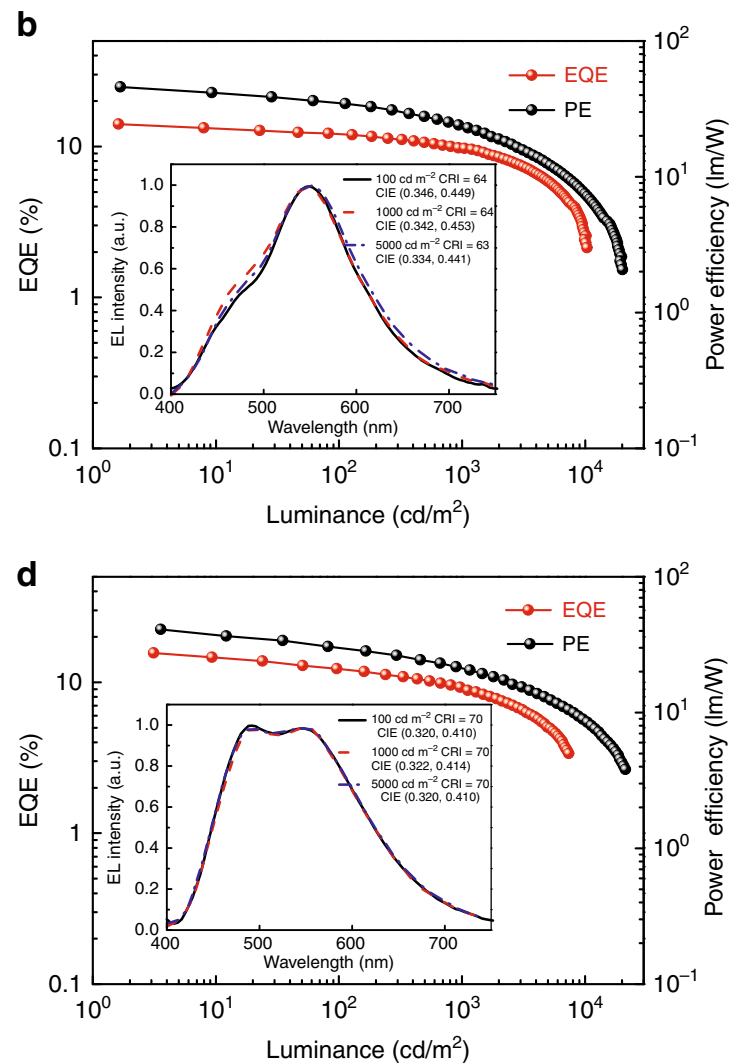

Fig. 3 Electroluminescence performance of reference devices. a Current density-voltage-luminance $(J-V-L)$ characteristics of the reference white organic light-emitting diodes (WOLEDs) with the single-emissive layer. The inset illustrates the detailed device structure of the optimized reference fluorescent WOLEDs. b External quantum efficiency (EQE) and power efficiency (PE)-luminance characteristics of the fluorescent WOLEDs. Inset exhibits the normalized electroluminescence spectra at the different luminance of 100,1000 , and $5000 \mathrm{~cd} / \mathrm{m}^{2}$, and the respective Commission Internationale de $L^{\prime} E$ clairage $(\mathrm{CIE})$ and color rendering index (CRI). c J-V-L characteristics of the reference WOLED with the double-emissive layer. The inset illustrates the detailed device structure of the optimized reference fluorescent WOLEDs. $\mathbf{d}$ EQE and PE-luminance characteristics of the fluorescent WOLEDs. Inset exhibits the normalized electroluminescence spectra at different luminance, and the respective CIE and CRI

device is almost sixfold longer than that of single W1 and nearly fivefold times longer than that of double W2, which can be attributed to more efficient exciton utilization and mitigated exciton quenching in a broadened emissive layer compared with the reference conventional devices.

Remarkably, according to our device design strategy, the performance of the optimized WOLEDs in this work significantly exceeds that of other reported all-fluorescent WOLEDs, especially for the PEs that are strongly enhanced. Supplementary Table 1 summaries the EL performances (without any out-coupling techniques) of representative fluorescent WOLEDs reported. However, like most TADF-based WOLEDs, the efficiencies still have much room that can be further improved compared with the state-of-the-art phosphorescent WOLEDs. We also notice that the orange TADF emitter in this work is not efficient enough because the photoluminescence quantum yield has not reached unity. To fully exploit the potential of our design concept, the introduction of efficient and stable ambipolar host materials for blue TADF emitters and more efficient orange/red TADF molecules with shorter excitation-state lifetime would be helpful.

Working mechanism. To study the working mechanism in the sensitized system, the transient photoluminescence (PL) behaviors and charge carrier-trapping effect were examined (see Fig. 4). We tested the transient PL of films consisting of DPEPO:30\% DMAC-DPS (film 1), DPEPO:30\% DMACDPS:0.5\% FDQPXZ (film 2), DPEPO:30\% DMAC-DPS: $1 \%$ FDQPXZ (film 3). Clearly the film 1 illustrates obvious prompt 

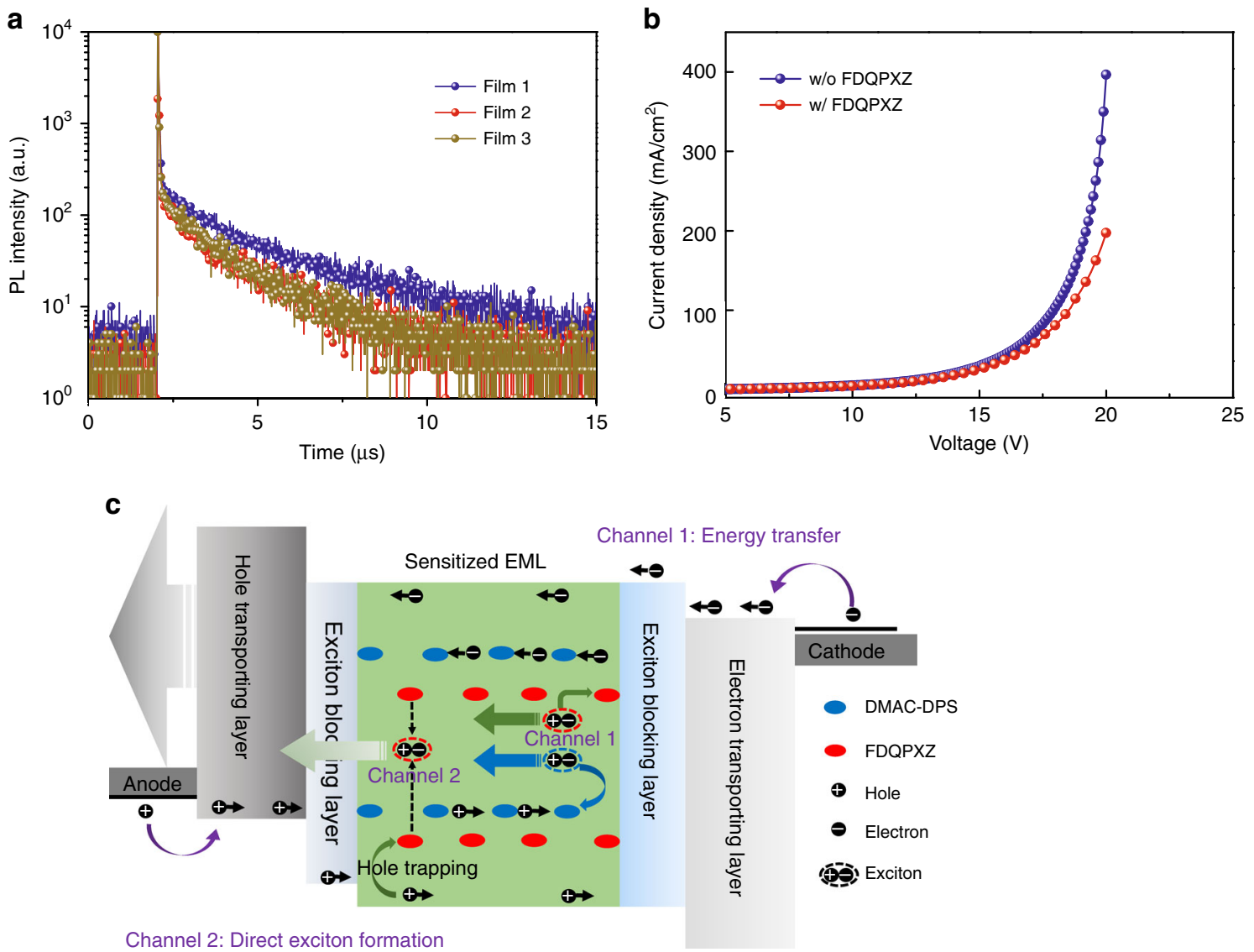

Fig. 4 Work mechanism of the proposed devices. a Transient photoluminescence decay curves measured at $470 \mathrm{~nm}$ with an excitation wavelength of $350 \mathrm{~nm}$. Film 1 consists of DPEPO:30\% DMAC-DPS (20 nm); film 2 is DPEPO:30\% DMAC-DPS:0.5\% FDQPXZ (20 nm); film 3 is DPEPO:30\% DMAC-DPS:1\% FDQPXZ. b Current density-voltage $(J-V)$ characteristics of the hole-only device with the structure of ITO/HATCN (10 nm)/TAPC: HATCN (20 wt $\%, 50 \mathrm{~nm}) /$ TAPC (20 nm)/DPEPO:25\% DMAC-DPS (20 nm) or DPEPO:25\% DMAC-DPS:1.0\% FDQPXZ (20 nm)/TAPC (20 nm)/TAPC: HATCN $(20 \mathrm{wt} \%, 50 \mathrm{~nm}) / \mathrm{HATCN}(10 \mathrm{~nm}) / \mathrm{Al}$. c Illustrated schematics of the work mechanism consisting of two parallel channels: efficient energy transfer and direct exciton formation process

and delayed parts in the PL decay curves, which is the classical characteristics of TADF (see Fig. 4a). When the orange TADF emitter doped into the blue-emitting layer, the lifetime and ratio of the delayed part of DMAC-DPS obviously decrease because of the efficient energy-transfer process from DMAC-DPS molecules to FDQPXZ, exhibiting the effect of orange TADF emitter sensitized by the high-energy-level blue TADF. Furthermore, to study the charge carrier-trapping effect of the orange TADF emitter in the system, we fabricated hole-only devices with the structure of ITO/HATCN $(10 \mathrm{~nm}) /$ TAPC: $20 \mathrm{wt} \%$ HATCN $(50$ $\mathrm{nm}) / \mathrm{TAPC}(20 \mathrm{~nm}) / \mathrm{DPEPO}: 25 \mathrm{wt} \%$ DMAC-DPS $(20 \mathrm{~nm})$ or DPEPO:25 wt $\%$ DMAC-DPS:1.0 wt\% FDQPXZ $(20 \mathrm{~nm}) /$ TAPC $(20 \mathrm{~nm}) / \mathrm{TAPC}: 20 \mathrm{wt} \%$ HATCN $(50 \mathrm{~nm}) / \mathrm{HATCN}(10 \mathrm{~nm}) / \mathrm{Al}$. When doping the orange FDQPXZ emitter into the DPEPO:25\% DMAC-DPS layer, the hole current decreases obviously, indicating that FDQPXZ molecule possesses the effective hole carriertrapping effect. This trapping effect is caused by the very large HOMO offset of $0.9 \mathrm{eV}$ between the DMAC-DPS/DPEPO and FDQPXZ molecules, which will be beneficial for improving the performance because it can decrease the polaron density and thus reduce the exciton-polaron quenching especially at the low current density ${ }^{7,9,12}$. Thus, in the sensitized system, there are two parallel working mechanisms, one is the efficient energy transfer from the blue DMAC-DPS to orange FDQPXZ, the other is the charge carrier trapping of orange-emitter molecules. These two parallel channels cooperatively let the present WOLED utilize all of the generated singlet and triplet excitons within the exciton recombination zone.

Optical simulation. Due to the determination of EQE parameters and the implementation of optical effects in a microcavity model, all individual light loss channels in OLEDs could be quantitatively demonstrated in detail ${ }^{44,45}$. Here, we provide a detailed optical simulation for our optimized WOLED to prove the nearly unitary exciton utilization efficiency. In general, to model the emissive characteristics of an OLED, it is assumed that the emissive zone contains an ensemble of mutually incoherent dipole radiators with distributions in the dipole orientations, locations, and frequencies. The electrical losses are ignored and the nonradiative losses exhibit only small variation because of the limited Purcell effect for highly efficient emitting materials ${ }^{46}$.

The detailed simulation parameters and process are also depicted in the Supplementary Figs. 3-5 and Supplementary Note 1 . The radiation characteristics of OLEDs are then obtained by the contributions over these distributions. Accordingly, Fig. 5a illustrates the relative photon distribution, i.e., the distributed quantum efficiencies of all channels in dependence of the electron-transporting layer (ETL) thickness. A theoretical maximum outcoupled EQE of $21.7 \%$ at the thickness of $60 \mathrm{~nm}$ can be predicted in our device structure, which is very close to the actually obtained value $(20.5 \%)$. It is worth to note that around $24.0 \%$ of generated photons is restricted in the substrate mode, 

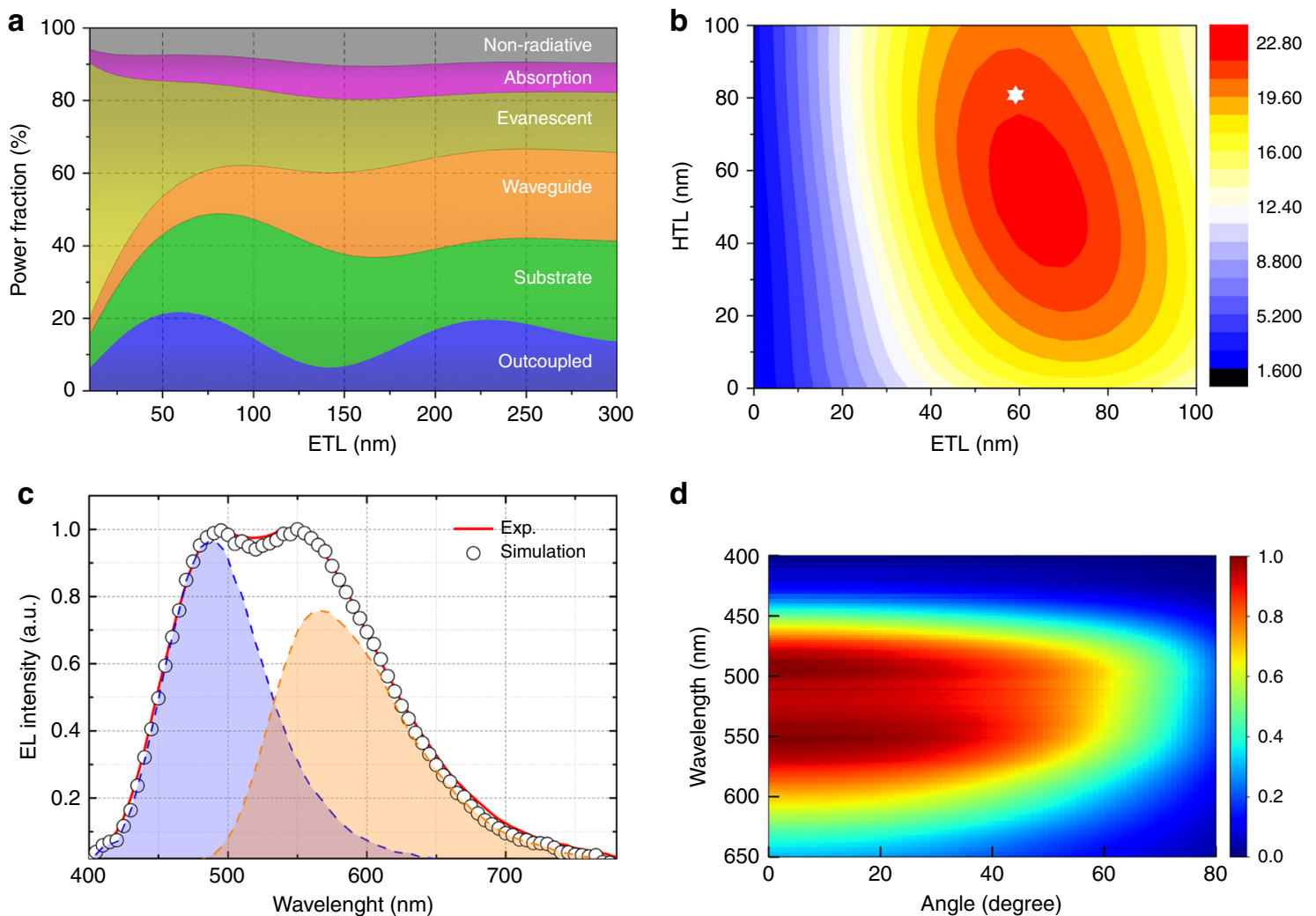

Fig. 5 Optical simulation. a Simulated photon distribution of all loss channels in dependence of the electron-transporting layer (ETL) thickness in the present white organic light-emitting diodes (WOLEDs). Most generated photons remain in waveguided and evanescent modes. b Calculated extraction efficiency from the simulated photon distribution of the present WOLEDs, HTL denotes the hole-transporting layer. The pentagram symbol represents the theoretical maximum external quantum efficiency (21.7\%) in our proposed structure. c The experimental and theoretical spectra of the WOLEDs according to the monochrome blue and orange spectra. $\mathbf{d}$ Angular resolved spectral radiant intensity (SRI) of the present WOLED with the electron-transporting layer thickness of $60 \mathrm{~nm}$ at the brightness of $1000 \mathrm{~cd} \mathrm{~m}^{-2}$. The displayed is the SRI in dependence of the wavelength $\lambda$ and polar angle $\theta$

and these photons could be extracted by simple out-coupling techniques such as the introduction of half sphere, indicating potentially $46.0 \% \mathrm{EQE}$ could be reached in our device structure. Figure $5 \mathrm{~b}$ shows the calculated coupling efficiency as a function of the hole transport and electron transport layer thickness. Without considering the electrical balance in real devices, a maximum value of $22.7 \%$ can be predicted at the ETL thickness of $65 \mathrm{~nm}$ and hole-transporting layer (HTL) of $50 \mathrm{~nm}$, which is also very close to the value of $21.7 \%$ at the ETL thickness of $60 \mathrm{~nm}$ in our electrically balanced device structure. Figure $5 \mathrm{c}$ gives the simulated spectrum at perpendicular direction with the photon contribution from blue and orange of 46 and 54\%, respectively, agreeing with experiment results. Figure $5 \mathrm{~d}$ describes the angledistributed spectra. The spectra are also quite stable with the angle changing, promising for large angle demanding application (see Supplementary Fig. 6). From these simulated results, it is believed that nearly unity exciton utilization efficiency has been realized in our proposed emissive layer architecture.

Exciton distribution. As seen, in the single-emissive W1 device, the blue emission intensity is low compared with the orange emission. The blue emission is effectively quenched by the orange emitter, but there is no happening in our present device. This effect should be due to following reasons: first, we broadened the exciton recombination zone, and meanwhile decreased the doping concentration of the orange emitter (FDQPXZ) and added the concentration of blue emitter (DMAC-DPS), suppressing the quenching effect. Second, we used the effective exciton-confined structure, the excitons can be effectively confined in the present device, but in a single W1 device the generated excitons in emissive layer will leak into the adjacent holetransporting layer because of the high triplet and singlet energy level of DMAC-DPS, which has been proved by the experiment of inserting sensing materials. Third, we introduced the efficient sensitized effect, the generated nonradiative triplet excitons will be converted into the radiative singlet excitons by the efficient reverse intercrossing process of DMAC-DPS. To prove these, we studied the exciton recombination distribution in the emitting layer of our present WOLED and the reference single W1. The detailed information has been shown in the Supplementary Information (see Supplementary Figs. 7-11 and Supplementary Notes 2, 3).

\section{Discussion}

Here, we demonstrate a universal approach to significantly improve the EL efficiencies of all-fluorescent WOLEDs based on TADF emitters. This design strategy can improve the charge balance, broaden the exciton recombination zone, and achieve the extreme utilization of singlet and triplet excitons within the EML, rendering extremely high efficiency together with good color stability, indicating their great potential for commercialization. The optical simulation shows the nearly unity exciton utilization efficiency. It is believed that our device design strategy can provide a new avenue for realizing high-performance all-fluorescent WOLEDs using TADF emitters. 


\section{Methods}

Fabrication process of WOLEDs. Clean glass substrates pre-coated indium tin oxide (ITO) with a sheet resistance of $10 \Omega$ per square can be commercially bought, and all devices are fabricated on these substrates. First, the ITO surface is cleaned using the detergents, and washed out with the deionized water. Then they are transferred into an oven for being dried at $120^{\circ} \mathrm{C}$ for $4 \mathrm{~h}$. After that, these substrates are treated with ultraviolet plasma for $20 \mathrm{~min}$ to decrease the ITO work function, then loaded into a deposition chamber. All functional layers are thermally evaporated grown in succession without breaking the vacuum $\left(\sim 5 \times 10^{-6} \mathrm{mbar}\right)$. The detailed typical evaporated rates of the organic materials, $\mathrm{Li}_{2} \mathrm{CO}_{3}$, and $\mathrm{Al}$ are $1-2,0.1-0.2$, and $10-20 \AA / s$, respectively. The effective emissive area was up to $0.4 \times 0.4 \mathrm{~cm}^{2}$, determined by the overlap between the Al and ITO electrodes.

Electroluminescence performance characteristics of WOLEDs. Keithley source measurement unit (Keithley 2400 and Keithley 2000) combined with a calibrated silicon photodiode are used for measuring the current-voltage-brightness characteristics. The external quantum efficiency is calculated from the EL spectra, luminance and current density assuming a Lambertian distribution. All above EL performance measurements are carried out at room temperature under ambient conditions. The device lifetime measurement is performed in the glovebox filled with $\mathrm{N}_{2}$ at room temperature.

Transient photoluminescence (PL) measurement. The measured films are grown on the quartz substrates. Edinburg FLS920 as the transient spectrometer and a picosecond pulsed UV-LASTER (LASTER 377) as the excitation source are used for the transient PL decay.

\section{Data availability}

The data that support the findings of this study are available from the corresponding author upon reasonable request.

Received: 18 January 2019 Accepted: 15 April 2019

Published online: 30 May 2019

\section{References}

1. Kido, J., Kimura, M. \& Nagai, K. Multilayer white light-emitting organic electroluminescent device. Science 267, 1332-1334 (1995).

2. Reineke, S., Thomschke, S. M., Lüssem, B. \& Leo, K. White organic lightemitting diodes: status and perspective. Rev. Mod. Phys. 85, 1245-1293 (2013).

3. Wu, Z. \& Ma, D. Recent advances in white organic light-emitting diodes. Mater. Sci. Eng. R. 107, 1-42 (2016).

4. Schwartz, G., Pfeiffer, M., Reineke, S., Walzer, K. \& Leo, K. Harvesting triplet excitons from fluorescent blue emitters in white organic light-emitting diodes. Adv. Mater. 19, 3672-3676 (2007).

5. Lai, C. C. et al. $m$-Indolocarbazole derivative as a universal host material for RGB and white phosphorescent OLEDs. Adv. Funct. Mater. 25, 5548-5556 (2015).

6. Ye, J. et al. Management of singlet and triplet excitons in a single emission layer: a simple approach for a high-efficiency fluorescence/phosphorescence hybrid white organic light-emitting device. Adv. Mater. 24, 3410-3014 (2012).

7. Wang, Q. \& Ma, D. Management of charges and excitons for highperformance white organic light-emitting diodes. Chem. Soc. Rev. 39, 2387-2398 (2010).

8. Liu, Y. et al. Simplified hybrid white organic light-emitting diodes with a mixed fluorescent blue emitting layer for exciton managing and lifetime improving. Adv. Opt. Mater. 4, 2051-2056 (2016).

9. Sun, N. et al. High-performance hybrid white organic light-emitting devices without interlayer between fluorescent and phosphorescent emissive regions. Adv. Mater. 26, 1617-1621 (2014).

10. Liu, X. K. et al. Remanagement of singlet and triplet excitons in single emissive-layer hybrid white organic light-emitting devices using thermally activated delayed fluorescent blue exciplex. Adv. Mater. 27, 7079-7085 (2015).

11. $\mathrm{Wu}, \mathrm{Z}$. et al. Precise exciton allocation for highly efficient white organic lightemitting diodes with low efficiency roll-off based on blue thermally activated delayed fluorescent exciplex emission. Adv. Optical Mater. 5, 1700415 (2017).

12. Zhu, L., Wu, Z., Chen, J. \& Ma, D. Reduced efficiency roll-off in allphosphorescent white organic light-emitting diodes with an external quantum efficiency of over 20\%. J. Mater. Chem. C. 3, 3304-3310 (2015).

13. Kanno, H., Holmes, R. J., Sun, Y., Kena-Cohen, S. \& Forrest, S. R. White stacked electrophosphorescent organic light-emitting devices employing $\mathrm{MoO}_{3}$ as a charge-generation layer. Adv. Mater. 18, 339-342 (2006).

14. Lee, S., Shin, H. \& Kim, J. J. High-efficiency orange and tandem white organic light-emitting diodes using phosphorescent dyes with horizontally oriented emitting dipoles. Adv. Mater. 26, 5864-5868 (2014).
15. Krummacher, B. C. et al. Highly efficient white organic light-emitting diode. Appl. Phys. Lett. 88, 113506 (2006).

16. Su, S. J., Gonmori, E., Sasabe, H. \& Kido, J. Highly efficient organic blue-and white-light-emitting devices having a carrier- and exciton-confining structure for reduced efficiency roll-off. Adv. Mater. 20, 4189-4194 (2008).

17. Zhang, D. et al. Highly efficient hybrid warm white organic light-emitting diodes using a blue thermally activated delayed fluorescence emitter: exploiting the external heavy-atom effect. Light.: Sci. Appl. 4, e232 (2015).

18. Pope, M., Kallmann, H. P. \& Magnante, P. Electroluminescence in organic crystals. J. Chem. Phys. 38, 2042-2043 (1963).

19. Dias, F. B. et al. Triplet harvesting with $100 \%$ efficiency by way of thermally activated delayed fluorescence in charge transfer OLED emitters. Adv. Mater. 25, 3707-3714 (2013).

20. Reineke, S. et al. White organic light-emitting diodes with fluorescent tube efficiency. Nature 459, 234-238 (2009).

21. Sun, Y. R. et al. Management of singlet and triplet excitons for efficient white organic light-emitting devices. Nature 440, 908-912 (2006).

22. Schwartz, G., Reineke, S., Rosenow, T. C., Walzer, K. \& Leo, K. Triplet harvesting in hybrid white organic light-emitting diodes. Adv. Funct. Mater. 19, 1319-1333 (2009).

23. Zheng, C.-J. et al. Novel efficient blue fluorophors with small singlet-triplet splitting: hosts for highly efficient fluorescence and phosphorescence hybrid WOLEDs with simplified structure. Adv. Mater. 25, 2205-2211 (2013).

24. Baldo, M. A. et al. Highly efficient phosphorescent emission from organic electroluminescent devices. Nature 395, 151-154 (1998).

25. Yersin, H., Rausch, A. F., Czerwieniec, R., Hofbeck, T. \& Fischer, T. The triplet state of organo-transition metal compounds: triplet harvesting and singlet harvesting for efficient OLEDs. Coord. Chem. Rev. 255, 2622-2652 (2011).

26. Uoyama, H., Goushi, K., Shizu, K., Nomura, H. \& Adachi, C. Highly efficient organic light-emitting diodes from delayed fluorescence. Nature 492, 234-238 (2012).

27. Cho, Y. J., Jeon, S. K., Chin, B. D., Yu, E. \& Lee, J. Y. The design of dual emitting cores for green thermally activated delayed fluorescent materials. Angew. Chem. Int. Ed. 54, 5201-5204 (2015).

28. Li, J. et al. Highly efficient organic light-emitting diode based on a hidden thermally activated delayed fluorescence channel in a heptazine derivative. Adv. Mater. 25, 3319-3323 (2013).

29. Zhang, D. et al. High-efficiency fluorescent organic light-emitting devices using sensitizing hosts with a small singlet-triplet exchange energy. $A d v$. Mater. 26, 5050-5055 (2014).

30. Nakanotani, H. et al. High-efficiency organic light-emitting diodes with fluorescent emitters. Nat. Commun. 5, 4016-4023 (2014).

31. Nakanotani, H., Masui, K., Nishide, J., Shibata, T. \& Adachi, C. Promising operational stability of high-efficiency organic light-emitting diodes based on thermally activated delayed fluorescence. Sci. Rep. 3, 2127 (2013).

32. Zhang, D., Cai, M., Zhang, Y., Zhang, D. \& Duan, L. Sterically shielded blue thermally activated delayed fluorescence emitters with improved efficiency and stability. Mater. Horiz. 3, 145-151 (2016).

33. Cho, Y. J., Yook, K. S. \& Lee, J. Y. Cool and warm hybrid white organic lightemitting diode with blue delayed fluorescent emitter both as blue emitter and triplet host. Sci. Rep. 5, 7859 (2015).

34. Lee, S. Y., Yasuda, T., Yang, Y. S., Zhang, Q. \& Adachi, C. Luminous butterflies: efficient exciton harvesting by benzophenone derivatives for fullcolor delayed fluorescence OLEDs. Angew. Chem. Int. Ed. 53, 6402-6406 (2014).

35. Higuchi, T., Nakanotani, H. \& Adachi, C. High-efficiency white organic lightemitting diodes based on a blue thermally activated delayed fluorescent emitter combined with green and red fluorescent emitters. Adv. Mater. 27, 2019-2023 (2015)

36. Zhao, B. et al. Highly efficient and color stable single-emitting-layer fluorescent WOLEDs with delayed fluorescent host. Org. Electron. 23, 208-212 (2015).

37. Song, W., Lee, I. \& Lee, J. Y. Host engineering for high quantum efficiency blue and white fluorescent organic light-emitting diodes. Adv. Mater. 27, 4358-4363 (2015)

38. Li, Y. et al. Design strategy of blue and yellow thermally activated delayed fluorescence emitters and their all-fluorescence white OLEDs with external quantum efficiency beyond 20\%. Adv. Funct. Mater. 26, 6904-6912 (2016).

39. Zhang, Q. et al. Efficient blue organic light-emitting diodes employing thermally activated delayed fluorescence. Nat. Photonics 8, 326-332 (2014).

40. Yu, L. et al. Achieving a balance between small singlet-triplet energy splitting and high fluorescence radiative rate in a quinoxaline-based orange-red thermally activated delayed fluorescence emitter. Chem. Commun. 52, 11012-11015 (2016)

41. Chang, Y. L. et al. Highly efficient warm white organic light-emitting diodes by triplet exciton conversion. Adv. Funct. Mater. 23, 705-712 (2013). 
42. Zhang, Y., Lee, J. \& Forrest, S. R. Tenfold increase in the lifetime of blue phosphorescent organic light-emitting diodes. Nat. Commun. 5, 5008 (2014).

43. Lee, J. et al. Hot excited state management for long-lived blue phosphorescent organic light-emitting diodes. Nat. Commun. 8, 15566 (2017).

44. Will, P. A. et al. Scattering quantified: Evaluation of corrugation induced outcoupling concepts in organic light-emitting diodes. Org. Electron. 58, 250-256 (2018).

45. Huang, Y. H. et al. Unlocking the full potential of conducting polymers for highefficiency organic light-emitting devices. Adv. Mater. 27, 929-934 (2015).

46. Meerheim, R., Furno, M., Hofmann, S., Lüssem, B. \& Leo, K. Quantification of energy loss mechanisms in organic light-emitting diodes. Appl. Phys. Lett. 97, 253305 (2010).

\section{Acknowledgements}

The authors gratefully acknowledge the National Key Research and Development Plan of China (2016YFB0400701), the National Natural Science Foundation of China (91833304, 21788102, 11661131001), and Guangzhou Science \& Technology plan project (201707020040) for the support of this research. Y.L. acknowledges financial support from China Scholarship Council (No. 201506920047). Z.W. appreciates the funding from the Alexander von Humboldt-Foundation.

\section{Author contributions}

Z.W. and D.M. conceived and designed the experiments. Z.W. carried out the device fabrication and characterization. L.Y. made the transient PL measurement. Z.W. and Y.L. made the optical simulation. Z.W., Y.L., C.Z., X.Q., D.Y., J.C., C.Y., H.K., K.L., and D.M. analyzed the data and co-wrote the paper. K.L. and D.M. supervised this project. All authors commented on the paper.

\section{Additional information}

Supplementary Information accompanies this paper at https://doi.org/10.1038/s41467019-10104-4.

Competing interests: The authors declare no competing interests.

Reprints and permission information is available online at http://npg.nature.com/ reprintsandpermissions/

Journal peer review information: Nature Communications thanks Franky So and the other, anonymous, reviewer(s) for their contribution to the peer review of this work.

Publisher's note: Springer Nature remains neutral with regard to jurisdictional claims in published maps and institutional affiliations.

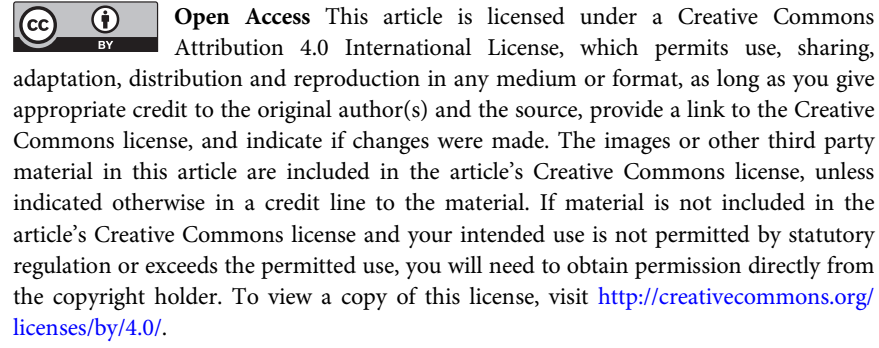

(C) The Author(s) 2019 